\title{
A Novel Method on Min-Max Limit Protection for Aircraft Engine Control
}

\author{
Wenjun Shu', Bing $\mathrm{Yu}^{1}$ and Hongwei $\mathrm{Ke}^{1}$ \\ ${ }^{1}$ Nanjing University of Aeronautics \& Astronautics, college of energy and power engineering, 29 Yudao Street, Nanjing, China
}

\begin{abstract}
Min-Max selector structure is widely employed in current aircraft engine control logic. And the structure must provide desired thrust and prevent the engine from exceeding any safety or operational limits. In this paper, a new control scheme, that is Min-Max selector structure composed of Sliding Mode (SM) regulator and linear regulator, is presented. The main regulator is a linear regulator and all limit regulators are SM regulators. It could overcome the possibility of limit violation for the traditional Min-Max, and don' $t$ need the augmented state references that is one drawback of SMC Min-Max(all regulators are SM regulator ). The simulation results show that the proposed approach could effectively prevent limit violation and can improve Min-Max limit protection for aircraft engine control.
\end{abstract}

\section{Introduction}

During the tracking process of the aircraft engine control, it must be ensured that all the constrained output variables (such as temperature, surge margin, speed and pressure, etc.) should be within the allowable working range. Otherwise, it will affect the life of the engine or even cause appearance of the undesirable characteristics, which will cause the failure of the engine and a series of serious consequences[1,2]. Therefore, how to play the best performance of aircraft engine under the limit protection becomes the key of its control research[3].

Traditional aircraft engine control system is usually controlled by the linear regulator using the Min-Max structure to achieve the constraint management[4-6]. In recent years, researches show that the traditional MinMax linear control structure has certain conservatism in the constraint treatment, which hinders the dynamic response that may be faster under the constraint. Although Min-Max algorithm is known as a methodology to control output constrained systems, this does not imply that limited outputs remain within their limits during transient state, and therefore, limit violation may occur for some variables[7, 8]. Research conducted by Ritcher et al. $[8,9]$ indicated that many shortcomings of the standard min-max approach can be removed by replacing linear regulators with Sliding Mode Control (SMC). And in this paper, it is called SMC Min-Max. The constraint outputs are able to work closely in the transient process. And it improves the dynamic response performance of the system. However, this technique requires the specification of augmented state references, which includes target steady values for the actuators, since integral controls used. The steady map of the nonlinear engine must be used to pre-calculate such references.
This drawback is aggravated when health parameter changes occur, since the steady map depends on these uncertain parameters. Ryan et al.[7] introduced the concept of using Conditionally Active (CA) limit regulators in the Min-Max architecture of typical engine control laws. The CA architecture in the Min-Max scheme only activates the limit regulators when the operating point is within a certain bound of the limit and approaching the limit at a faster rate than the prescribed one. Imani et al.[10] presented a strategy to design linear regulators of Min-Max selector control to improve transient limit protection by using Linear Matrix Inequalities (LMI) approach. It decreases the possibility of transient limit violation and can be considered as an effective approach to improve Min-Max limit protection in aircraft engine control. But the strategy did not show an advantage in response speed.

In this paper, a Min-Max structure controller composed of linear regulator and SM regulator is used for aircraft turbofan engine, whose architecture is shown as Fig. 1. The main regulator is a linear regulator while all the limit regulators are SM regulators. The proposed approach don't need state references and the SM limit regulators will improve the response speed without the risk of exceeding the physical or safety limits.

\section{The improvement of Min-Max controller}

The turbofan engine is frequently controlled by a feedback loop where fuel flow rate is the control input and fan speed is the sensed variable. In this paper, a twospool turbofan engine model in the $90,000 \mathrm{lb}$. thrust class, linearized at an altitude of 25,000 ft. and Mach number 0.62 is considered. And it is obtained from the 
Commercial Modular Aeropropulsion System Simulation (C-MAPSS)[10, 11]. Table 1 shows some output variables and their units. The linear model is shown as follows:
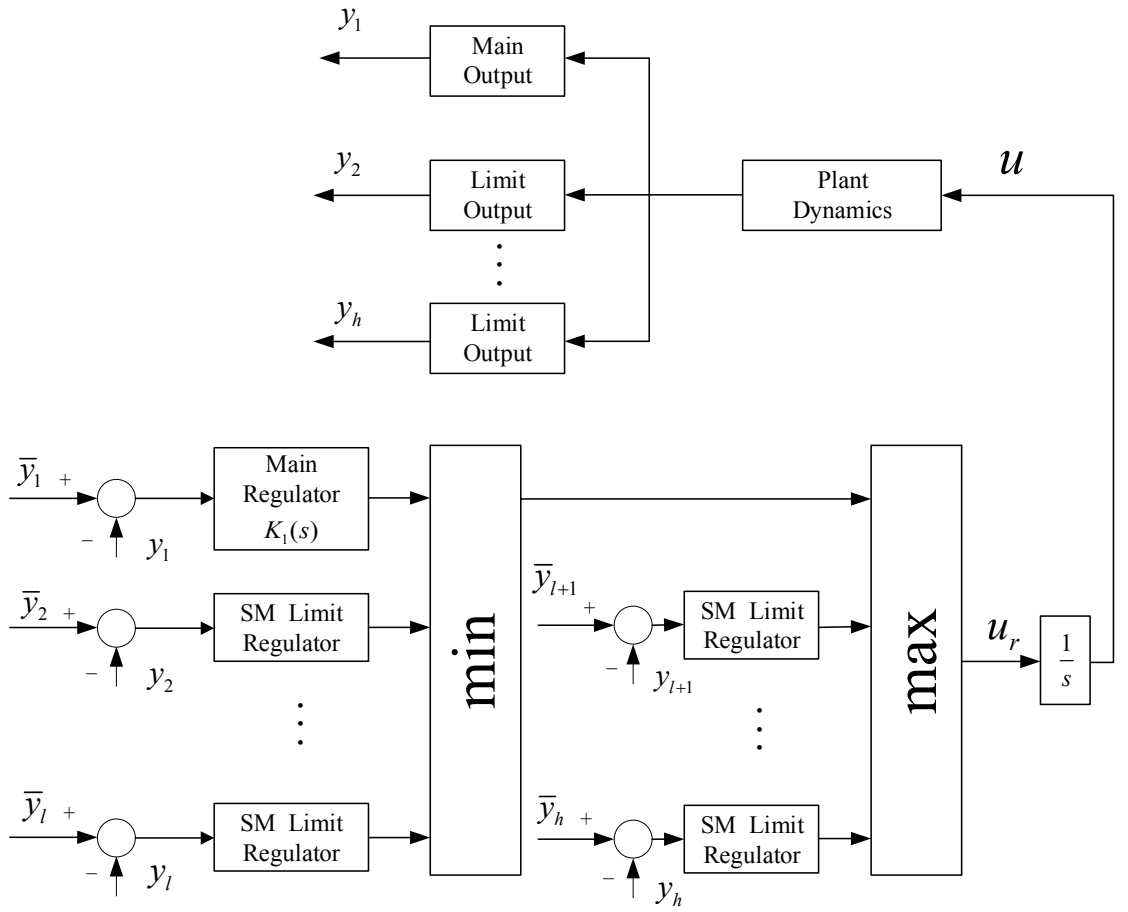

Figure 1. The new Min-Max multi-regulator system with integral control.

$$
\begin{gathered}
\dot{\boldsymbol{x}}=\boldsymbol{A} \boldsymbol{x}+\boldsymbol{B} u \\
\dot{u}=u_{r} \\
y_{i}=\boldsymbol{G}_{i} \boldsymbol{x}+\theta_{i} u
\end{gathered}
$$

Where $\boldsymbol{x}=\left[\begin{array}{ll}\Delta N_{f} & \Delta N_{c}\end{array}\right]^{T}$ is state variables vector with fan speed increment and core speed increment, $u=$ $\Delta W_{f}$ is fuel flow increment as control input, and $y_{i}$, for $\mathrm{i}$ $=2,3,4,5$ are output increments. The model matrices come from references[10, 11].

Table 1. Some output variables of aircraft engine.

\begin{tabular}{|l|l|}
\hline Variables & Units \\
\hline Fan speed, $N_{f}$ & $\mathrm{rpm}$ \\
\hline Core speed, $N_{c}$ & $\mathrm{rpm}$ \\
\hline Fuel flow rate, $W_{f}$ & $\mathrm{pps}$ \\
\hline High-pressure compressor stall margin, $S m H P C$ & $\%$ \\
\hline High-pressure turbine outlet temperature, $T_{48}$ & ${ }^{\circ} R$ \\
\hline Engine pressure ratio, $E P R$ & - \\
\hline High-pressure compressor outlet pressure, $P_{S 30}$ & $\mathrm{psia}$ \\
\hline
\end{tabular}

The control objective is to follow the desired fan speed while maintaining constrained outputs within bounds at all times. The outputs and their incremental limits come from references[10, 11].
SMC Min-Max requires the specification of augmented state references, which includes target steady values for the actuators. However, that real-time implementations of the SM limit regulators do not require state references, due to the definition of the sliding function. Only the limit setting and a real-time output measurement are needed to calculate the sliding function[9]. To overcome this shortcoming, the main regulation is replaced by a linear regulator. Meanwhile, all limit regulations are SM regulators that will improve the response performance without exceeding the limits.

As shown in Fig.1, the linear regulator $K_{1}(s)$ is used as main regulator. Its parameter values come from the reference [10].

$$
K_{1}(s)=\frac{0.74 s+0.8}{s+40}
$$

In order to ensure that the limited output does not exceed its limit, SM limit regulators are used for all the limits. Due to space limitation, the method of SMC will not be repeated and just is given brief introduction[11]. The sliding function is defined by $s_{i}=y_{i}-\bar{y}_{l}(i=$ $2,3,4,5)$, and $\bar{y}_{l}$ is the physical or safety limit. The control law of SM limit regulator is given below in Eq. (3).

$$
u_{r i}=-\frac{1}{\Theta_{i}}\left(\boldsymbol{G}_{i}(\boldsymbol{A} \boldsymbol{x}+\boldsymbol{B} u)+\eta_{i} \operatorname{sgn}\left(s_{i}\right)\right)
$$

Where, $\eta_{i}$ is switching gain, and it directly affects dynamic response; $\operatorname{sgn}(\cdot)$ is saturation function. In this paper, the switch gain is set to $\eta_{i}=15\left|\theta_{i}\right|(i=2,3,4,5)$.

\section{Simulation results and discussion}


As the engine thrust cannot be sensed in a reliable way, fan speed variable is considered as thrust representative in this study. The performance is compared with the traditional Min-Max algorithm with controller and the LMI Min-Max controller. And all controllers are digital controllers with the sampling time of 0.015 seconds[6].

Fig.2 shows the system response in tracking the desired fan speed. Although settling time for both is three identical, rise time for the new controller is less than the LMI one and keeps the same as traditional one. Incremental fuel flow input has been shown in Fig. 3 for all controllers.

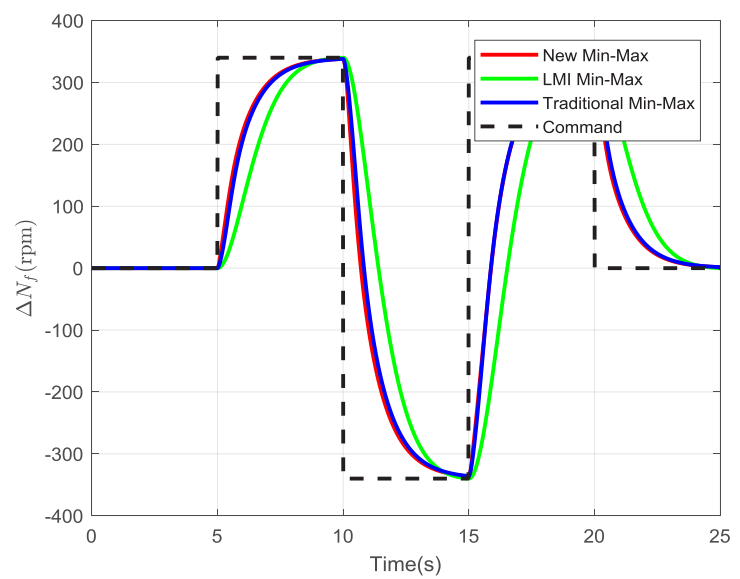

Figure 2. Fan speed response.

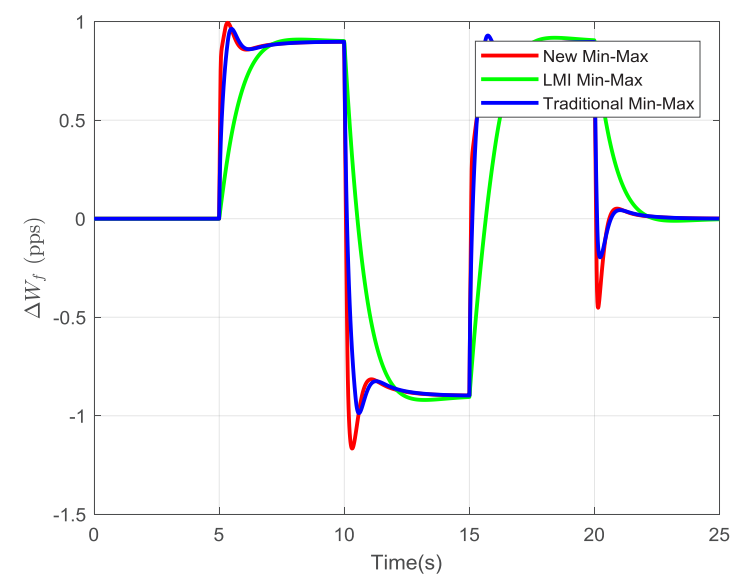

Figure 3. Fuel flow input.

Fig. 4 shows the output $\triangle \boldsymbol{S} \boldsymbol{m} \boldsymbol{H P C}$ and its detail with enlarged scale. Although rise time of new controller and traditional are same, it's obvious that the new proposed controller protects the constraint unlike the traditional. Fig. 5, Fig. 6 and Fig. 7 are the outputs of $\Delta \boldsymbol{T}_{48}, \Delta \boldsymbol{P}_{s 30}$ and $\triangle \boldsymbol{E} \boldsymbol{P} \boldsymbol{R}$. All controllers satisfy the constrains.

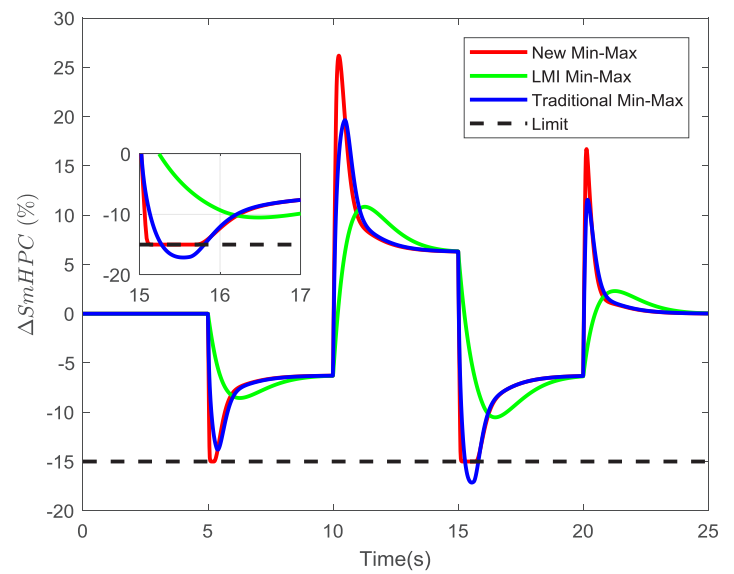

Figure 4. Lower limited output $\triangle \boldsymbol{S} \boldsymbol{M H} \boldsymbol{H P C}$.

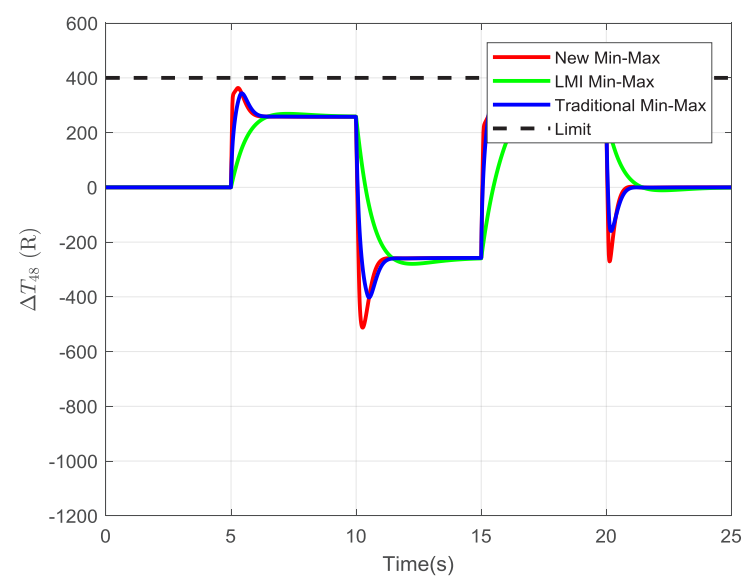

Figure 5. Upper limited output $\Delta \boldsymbol{T}_{\mathbf{4 8}}$.

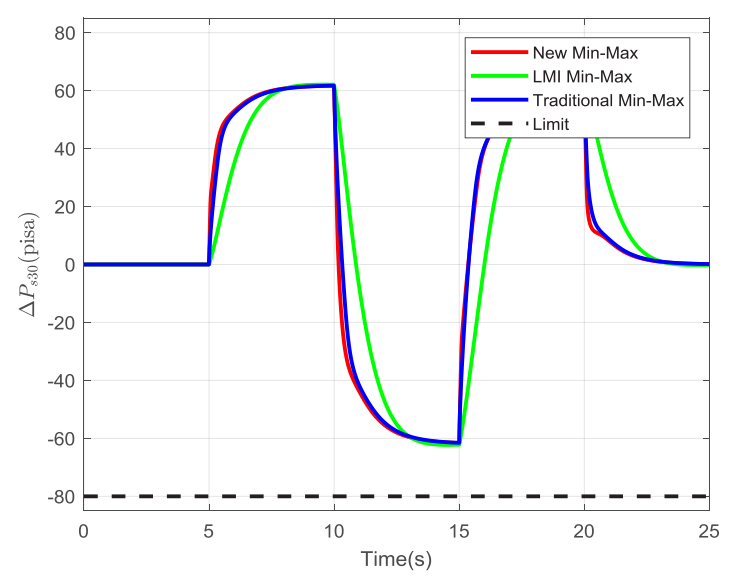

Figure 6. Lower limited output $\Delta \boldsymbol{P}_{\boldsymbol{s 3 0}}$. 


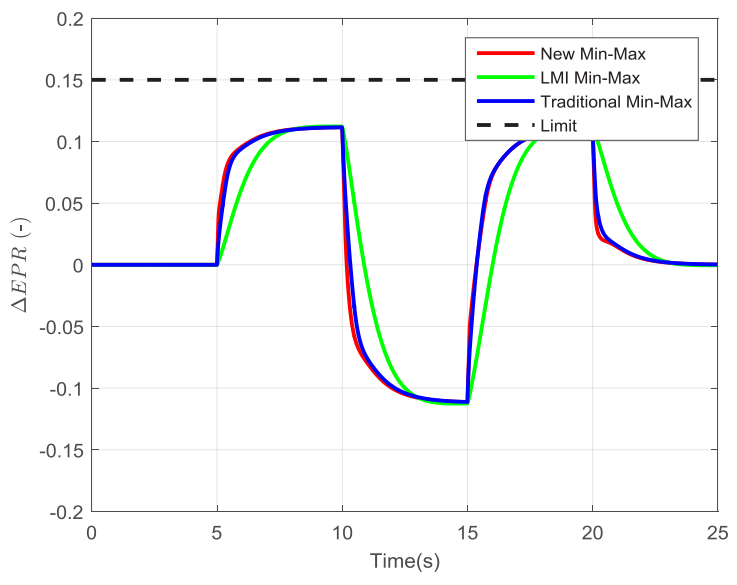

Figure 7. Upper limited output $\Delta \boldsymbol{E} \boldsymbol{P R}$.

Because the SM limit regulators are used, the constraint outputs are rapidly approaching the limit value in the response without exceeding the limit value. It's proved that the new controller has the potential to reach the reference value faster. However, all of the above simulation results can not well show this advantages. Now, the linear main regulator will be redesigned that is shown in follow:

$$
K_{1}(s)=\frac{0.74 s+1.8}{s+40}
$$

It adds a new comparison object that is SMC MinMax means all the regulators are SMC structure, and others are same as the previous simulation.

Fig. 8 shows the system response in tracking the desired fan speed after the main regulator redesign. The settling time for both of new Min-Max and SMC MinMax controller is almost identical, and both of them are faster than traditional and LMI Min-Max controller.

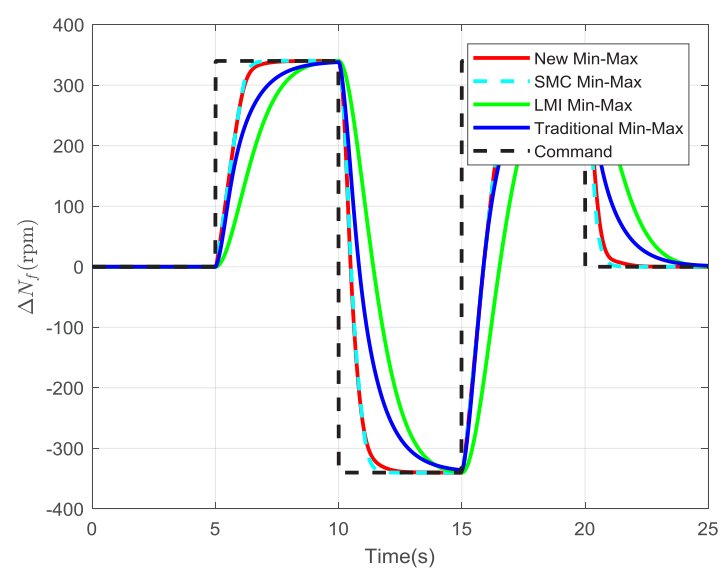

Figure 8. Fan speed response after the main regulator redesign.

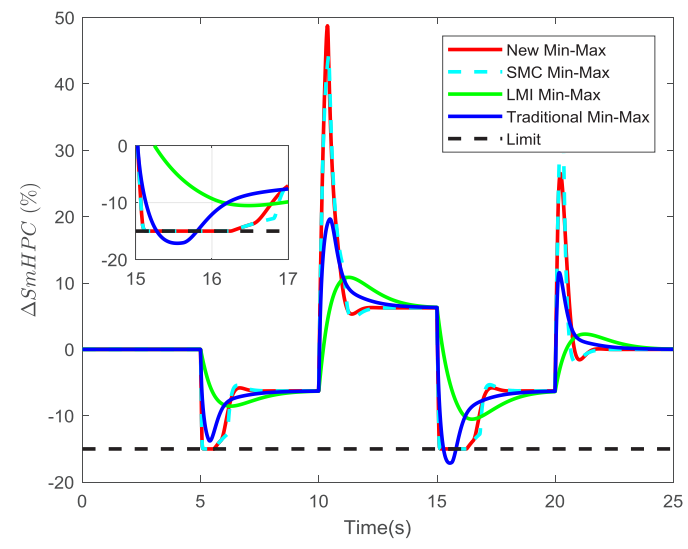

Figure 9. Lower limited output $\triangle \boldsymbol{S} \boldsymbol{m} \boldsymbol{H} \boldsymbol{P C}$ after the main regulator redesign.

Due to the space limit, the constraint output only $\triangle \boldsymbol{S m H P C}$ is shown in Fig. 9. And it demonstrates that all the controllers except the traditional Min-Max controller prevent limit violation.

\section{Conclusion}

In this paper, a new control scheme, that is Min-Max selector structure composedof Sliding Mode (SM) regulator and linear regulator, is presented. The main regulator is a linear regulator and all limit regulators are SM regulators. It overcomes the possibility of limit violation for the traditional Min-Max, and don't need the augmented state references which is one drawback of SMC Min-Max. The simulation results show that the proposed approach could effectively prevents limit violation and improve Min-Max limit protection in aircraft engine control.

\section{Acknowledgments}

This work was supported in part by the Natural Science Foundation of China under Grant 51406083, in part by the Fundamental ResearchFunds for the Central Universities under Grant NJ20160037, and in part by the Natural Science Foundation of Jiangsu Province underGrant BK20140820.

\section{References}

1. Jaw, L.C. and J.D. Mattingly, Aircraft engine controls : design, system analysis, and health monitoring. American Institute of Aeronautics and Astronautics (2009)

2. Du, X., Y.Q. Guo, and X.L. Chen, Limit management of aircraft engine based on nonlinear model predictive control method. Journal of Aerospace Power. 30(7): 1766-1771 (2015)

3. Csank, J.T. and R.D. May, A Sensitivity Study of Commercial Aircraft Engine Response for Emergency Situations: NASA/TM-2011-217004 (2011) 
4. May, R., et al., A High-Fidelity Simulation of a Generic Commercial Aircraft Engine and Controller: AIAA-2010-6630 (2010)

5. Iii, H.A.S. and H. Brown, Control of jet engines. Control Engineering Practice. 7(9): 1043-1059 (1999)

6. Csank, J., et al., Control Design for a Generic Commercial Aircraft Engine: AIAA-2010-6629 (2010)

7. May, R.D. and S. Garg, Reducing Conservatism in Aircraft Engine Response Using Conditionally Active Min-Max Limit Regulators: AIAA-2012217814 (2012)

8. Richter, H., Multiple Sliding Modes with Override Logic: Limit Management in Aircraft Engine Controls. J GUID CONTROL DYNAM. 35(4): 1132-1142 (2012)

9. Richter, H., Advanced Control of Turbofan Engines. Springer New York (2012)

10. Imani, A. and M. Montazeri-Gh, Improvement of Min-Max Limit Protection in Aircraft Engine Control: An LMI Approach. AEROSP SCI TECHNOL. 68 (2017)

11. Richter, H., A multi-regulator sliding mode control strategy for output-constrained systems. AUTOMATICA. 47(10): 2251-2259 (2011) 